\title{
Double-Diffusive of a Nanofluid in a Rectangle-Shape Mounted on a Cavity Saturated by Heterogeneous Porous Media
}

\author{
Abdelraheem M. Aly, ${ }^{1,2}$ Ehab Mohamed Mahmoud $\mathbb{D}^{3,4}$ Hijaz Ahmad (D), 5,6 \\ and Shao-Wen Yao ${ }^{7}{ }^{7}$ \\ ${ }^{1}$ Department of Mathematics, College of Science, King Khalid University, P.O. Box 9004, Abha 61413, Saudi Arabia \\ ${ }^{2}$ Department of Mathematics, Faculty of Science, South Valley University, Qena 83523, Egypt \\ ${ }^{3}$ Electrical Engineering Department, College of Engineering, Prince Sattam Bin Abdulaziz University, P.O. Box 11991, \\ Wadi Addwasir, Saudi Arabia \\ ${ }^{4}$ Electrical Engineering Department, Faculty of Engineering, Aswan University, P.O. Box 81542, Aswan, Egypt \\ ${ }^{5}$ Department of Basic Sciences, University of Engineering and Technology, Peshawar, Pakistan \\ ${ }^{6}$ Section of Mathematics, International Telematic University Uninettuno, Corso Vittorio Emanuele II 39, Roma 00186, Italy \\ ${ }^{7}$ School of Mathematics and Information Science, Henan Polytechnic University, Jiaozuo 454000, China
}

Correspondence should be addressed to Shao-Wen Yao; yaoshaowen@hpu.edu.cn

Received 6 December 2020; Revised 10 January 2021; Accepted 28 January 2021; Published 11 February 2021

Academic Editor: Riaz Ahmad

Copyright (C) 2021 Abdelraheem M. Aly et al. This is an open access article distributed under the Creative Commons Attribution License, which permits unrestricted use, distribution, and reproduction in any medium, provided the original work is properly cited.

\begin{abstract}
This study presents numerical simulations on double-diffusive flow of a nanofluid in two cavities connected with four vertical gates. Novel shape of an outer square shape mounted on a square cavity by four gates was used. Heterogeneous porous media and $\mathrm{Al}_{2} \mathrm{O}_{3}$-water nanofluid are filled in an inner cavity. Outer rectangle shape is filled with a nanofluid only, and its left walls carry high temperature $T_{h}$ and high concentration $C_{h}$. The right walls of a rectangle shape carry low temperature $T_{c}$ and low concentration $C_{c}$ and the other walls are adiabatic. An incompressible smoothed particle hydrodynamics (ISPH) method is applied for solving the governing equations of velocities, temperature, and concentration. Results are introduced for the effects of a buoyancy ratio $(-2 \leq N \leq 2)$, Darcy parameter $\left(10^{-3} \leq \mathrm{Da} \leq 10^{-5}\right)$, solid volume fraction $(0 \leq \phi \leq 0.05)$, and porous levels. Main results are indicated in which the buoyancy ratio parameter adjusts the directions of double-diffusive convection flow in an outer shape and inner cavity. Adding more concentration of nanoparticles reduces the flow speed and maximum of the velocity field. Due to the presence of a porous medium layer in an inner cavity, the Darcy parameter has slight changes inside the rectangle shape.
\end{abstract}

\section{Introduction}

Double-diffusive convection is a form of convection flow resulting from the variations of the diffusion rates [1]. Double-diffusive has several applications such as fuel cells, diffusion of chemical pollutants in ocean, and nuclear waste storage. In addition, heat and mass transfer in porous media are surviving in numerous engineering applications including oceanography, geophysics, chemical engineering, and thermal engineering [2]. Double-diffusive convection in an annulus has been studied intensively due to its applications in dying and cleaning operations and energy in solar ponds [3].
The adjoint influences of thermal and solutal buoyancy forces generate complex flow in a porous annulus. Beji et al. [4] utilized the Darcy model to investigate double-diffusive convection in a porous annulus. Double-diffusive convection in a porous annulus subjected to mass and heat fluxes was introduced by Marcoux et al. [5]. Nithiarasu et al. [6] generalized a model for non-Darcy flow of double-diffusive convection in a porous annulus. Lee et al. [7] examined the multilayer flow in double-diffusive convection of salt water filled in a rotating annulus. Chen et al. [8] adopted lattice Boltzmann model to investigate the double-diffusive in a vertical annulus by considering opposing gradients of the temperature and concentration. Goyeau [9] studied the 
double-diffusive convection in a porous cavity below Darcy number and porosity impacts. Chamkha and Al-Mudhaf [10] used the finite difference method to check the effects of an inclination angle and buoyancy ratio on double-diffusive in a tilted porous cavity.

In the recent years, nanofluid has many applications in solar energy systems, nuclear reactors, heat exchangers, and so on [11-19]. Bhatti et al. [20] discussed the impacts of chemical reaction and nonlinear thermal radiation on unsteady 3D boundary layer flow of a viscous nanofluid having gyrotactic microorganisms through a stretching porous cylinder. Mohebbi and Rashidi [21] used the Lattice Boltzmann Method (LBM) to study numerically the natural convection flow of nanofluid in $L$-shaped enclosure containing a heating obstacle. Chowdhury et al. [22] studied the double-diffusive convection in an enclosure filled by porous media and nanofluid with considering heat generation effects. The ISPH method was adopted by Aly and Raizah [23] to simulate double-diffusive convection in an enclosure filled with a nanofluid.

The topic of the fluid flow and heat transfer in heterogeneous porous media has many applications in remediation, enhanced oil recovery, and geological CO2 reservation. The porous media are heterogeneous porous media when the permeability varies from point to point in a medium [24-26].

Since the last decade, mesh-free methods became a wellalternative tool for overcoming disadvantage of mesh methods in simulating free-surface flows and high deformation problems. Lucy [27] and Gingold and Monaghan [28] firstly introduced smoothed particle hydrodynamics to simulate astrophysical problems. The smoothed particle hydrodynamics $(\mathrm{SPH})$ method is employed in several fields [29-35]. An incompressible SPH (ISPH) method has the ability for solving several complex problems by showing a good efficiency [36-42].

The SPH method is considered an excellent numerical method for the problems of a highly deforming surface/ interface such as free-surface flow, dam break, wave dynamics, impact flows, and bubble dynamics. In addition, the SPH method deals with interfaces naturally without any special requirements. The incompressible version of the SPH method entitled the ISPH method and has mesh-free advantages. The ISPH method can handle the doublediffusive convection flow in a complex cavity by an easy way.

In the current research, the ISPH method is used for the simulation of thermo-solutal convection in an outer square shape connected with a center square cavity. The outer square shape and inner square cavity are filled with $\mathrm{Al}_{2} \mathrm{O}_{3}$-water nanofluid, and the inner cavity is saturated by a heterogeneous porous medium. It is found that an augmentation in buoyancy ratio parameter increases the thermo-solutal convection in both of outer square shape and inner cavity. Moreover, the directions of thermo-solutal convection are strongly depending on the values of buoyancy ratio parameter. Presence of a porous medium in an inner square cavity only makes slight effects of Darcy parameter and porous medium levels in maximum velocity field. An incremental in solid volume fraction augments viscosity of water, and consequently, the flow speed decreases.

\section{Mathematical Formulations}

Figure 1 presents the initial physical model and initial particle distributions. In the current physical model, the outer square shape has a height $2 L$ and the width of a square shape equals 0.1 . The inner center cavity is a square cavity with a height $L$, and there are four normal gates between an outer square shape and an inner square cavity. Only the left walls of an outer square shape are carrying $T_{h}$ and $C_{h}$ and right walls have $T_{c}$ and $C_{c}$, and the other walls are adiabatic. The nanofluid occupied an outer square shape and inner square cavity, and the nanofluid is modeled by a one-phase model. Heterogeneous porous media are saturated inner square cavity only and the porous media are in thermal equilibrium with nanofluid. Thermophysical properties of water (base fluid) and $\mathrm{Al}_{2} \mathrm{O}_{3}$ at $T=310 \mathrm{~K}$ are introduced in Table 1.

Time dependent of double-diffusive convection for nanofluid flow according to $[43,44]$ is 


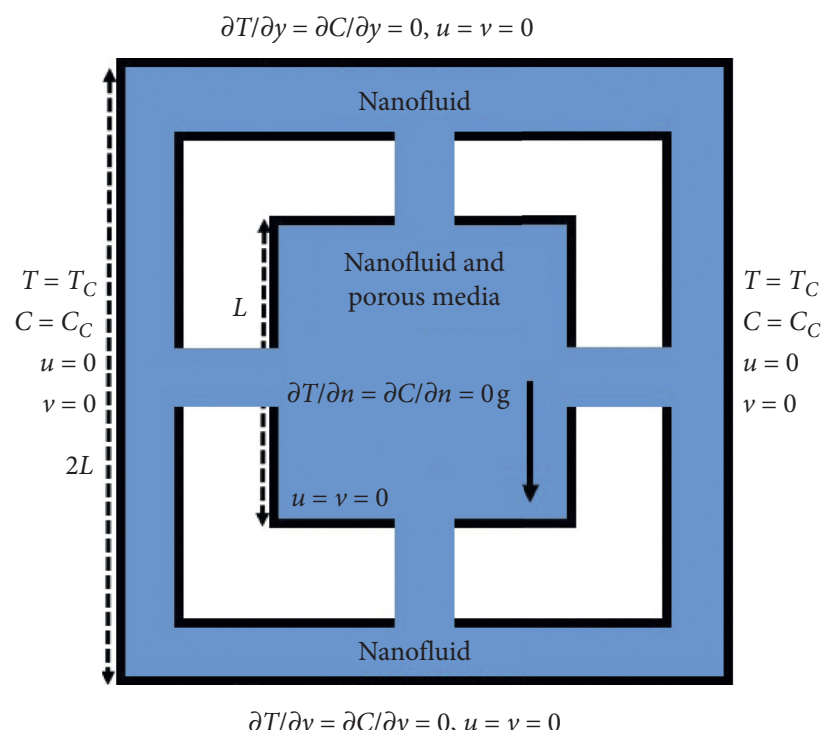

(a)

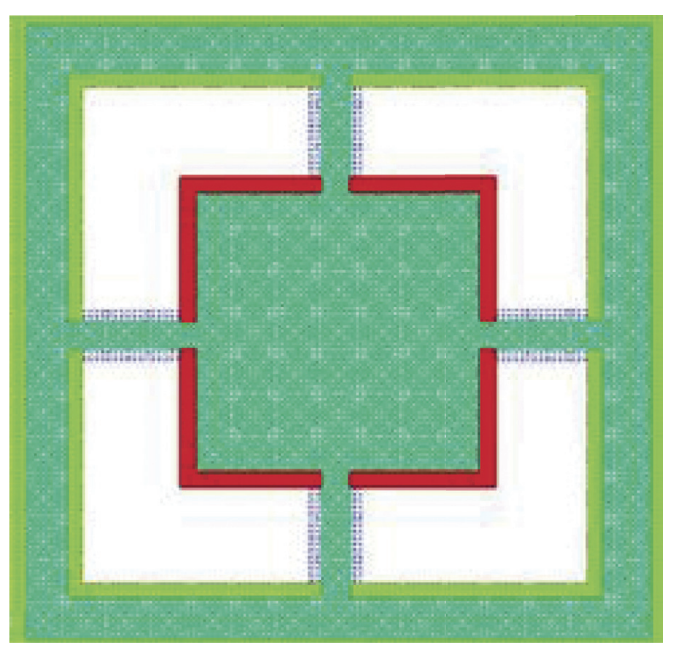

(b)

FIgURE 1: Initial schematic diagram and initial particle distributions.

TABLe 1: Nanofluid thermo-physical properties at $T=310 \mathrm{~K}$ [45-47].

\begin{tabular}{lccccc}
\hline & $\rho\left(\mathrm{Kgm}^{-3}\right)$ & $K(\mathrm{~W} / \mathrm{mK})$ & $C p(\mathrm{~J} / \mathrm{kgK})$ & $\beta \times 10^{-5} \mathrm{~K}^{-1}$ & $3(\mathrm{~nm})$ \\
\hline $\mathrm{H}_{2} \mathrm{O}$ & 993 & 0.628 & 4178 & 36.2 & 0.385 \\
$\mathrm{Al}_{2} \mathrm{O}_{3}$ & 3970 & 40 & 765 & 0.85 & 33 \\
\hline
\end{tabular}

$$
\frac{\partial U}{\partial X}+\frac{\partial V}{\partial Y}=0
$$

$$
\begin{aligned}
& \frac{\rho_{\mathrm{nf}}}{\rho_{f}}\left(\frac{1}{\varepsilon} \frac{\partial U}{\partial \tau}+\frac{U}{\varepsilon^{2}} \frac{\partial U}{\partial X}+\frac{V}{\varepsilon^{2}} \frac{\partial U}{\partial Y}\right) \\
= & -\frac{\rho_{\mathrm{nf}}}{\rho_{f}} \frac{\partial P}{\partial X}+\frac{1}{\varepsilon} \frac{\partial}{\partial X}\left(\operatorname{Pr} \frac{\mu_{\mathrm{nf}}}{\mu_{f}} \frac{\partial U}{\partial X}\right)+\frac{1}{\varepsilon} \frac{\partial}{\partial Y}\left(\operatorname{Pr} \frac{\mu_{\mathrm{nf}}}{\mu_{f}} \frac{\partial U}{\partial Y}\right)-\delta \frac{\operatorname{Pr} \mu_{\mathrm{eff}}}{\mu_{\mathrm{nf}} K^{*}(X, Y) \mathrm{Da}} U, \\
& \frac{\rho_{\mathrm{nf}}}{\rho_{f}}\left(\frac{1}{\varepsilon} \frac{\partial V}{\partial \tau}+\frac{U}{\varepsilon^{2}} \frac{\partial V}{\partial X}+\frac{V}{\varepsilon^{2}} \frac{\partial V}{\partial Y}\right) \\
= & -\frac{\rho_{\mathrm{nf}}}{\rho_{f}} \frac{\partial P}{\partial X}+\frac{1}{\varepsilon} \frac{\partial}{\partial X}\left(\operatorname{Pr} \frac{\mu_{\mathrm{nf}}}{\mu_{f}} \frac{\partial V}{\partial X}\right)+\frac{1}{\varepsilon} \frac{\partial}{\partial Y}\left(\operatorname{Pr} \frac{\mu_{\mathrm{nf}}}{\mu_{f}} \frac{\partial V}{\partial Y}\right)-\delta \frac{\operatorname{Pr} \mu_{\mathrm{eff}}}{\mu_{\mathrm{nf}} K^{*}(X, Y) \mathrm{Da}} V+\frac{(\rho \beta)_{\mathrm{nf}}}{(\rho \beta)_{f}} \operatorname{PrRa}(\theta+N \Phi),
\end{aligned}
$$

$$
\begin{aligned}
& \gamma \frac{\partial \theta}{\partial \tau}+U \frac{\partial \theta}{\partial X}+V \frac{\partial \theta}{\partial Y}=\frac{\left(\rho c_{p}\right)_{f}}{\left(\rho c_{p}\right)_{\mathrm{nf}}}\left[\frac{\partial}{\partial X}\left(\zeta(X, Y) \frac{\partial \theta}{\partial X}\right)+\frac{\partial}{\partial Y}\left(\zeta(X, Y) \frac{\partial \theta}{\partial Y}\right)\right] \\
& \frac{\partial \Phi}{\partial \tau}+\frac{U}{\varepsilon} \frac{\partial \Phi}{\partial X}+\frac{V}{\varepsilon} \frac{\partial \Phi}{\partial Y}=\frac{1}{\operatorname{Le}}\left[\frac{\partial}{\partial X}\left(\Pi(X, Y) \frac{\partial \Phi}{\partial X}\right)+\frac{\partial}{\partial Y}\left(\Pi(X, Y) \frac{\partial \Phi}{\partial Y}\right)\right] .
\end{aligned}
$$


The porous matrix expressions are

$$
\begin{aligned}
K^{*}(X, Y) & =e^{\eta_{1} H X+\eta_{2} H Y}, \\
\zeta(X, Y) & =(1-\varepsilon) e^{\eta_{1} H X+\eta_{2} H Y}+\varepsilon \frac{k_{\mathrm{nf}}}{k_{f}}, \\
\Pi(X, Y) & =\delta \frac{D_{\mathrm{eff}}(X, Y)}{D_{0}}=\delta \frac{D_{o} e^{\eta_{1} H X+\eta_{2} H Y}}{D_{0}}=\delta e^{\eta_{1} H X+\eta_{2} H Y}, \\
\gamma & =\frac{(1-\varepsilon)\left(\rho c_{p}\right)_{p}+\varepsilon\left(\rho c_{p}\right)_{\mathrm{nf}},}{\left(\rho c_{p}\right)_{\mathrm{nf}}}
\end{aligned}
$$

where

$$
\delta= \begin{cases}0,(\varepsilon=1.0), & \text { nanofluid only, } \\ 1,(\varepsilon=0.6), & \text { nanofluid and porous medium. }\end{cases}
$$

The expressions of a nanofluid according to [45-47] are

$$
\begin{aligned}
\rho_{\mathrm{nf}} & =\phi \rho_{p}+(1-\phi) \rho_{f}, \\
\alpha_{\mathrm{nf}} & =\frac{k_{\mathrm{nf}}}{\left(\rho c_{p}\right)_{\mathrm{nf}}}, \\
(\rho \beta)_{\mathrm{nf}} & =\phi(\rho \beta)_{p}+(1-\phi)(\rho \beta)_{f}, \\
\left(\rho c_{p}\right)_{\mathrm{nf}} & =\phi\left(\rho c_{p}\right)_{p}+(1-\phi)\left(\rho c_{p}\right)_{f}, \\
\mu_{\mathrm{nf}} & =\frac{\mu_{f}}{\left(1-34.87\left(d_{p} / d_{f}\right)^{-0.3} \varphi^{1.03}\right)^{\prime}}, \\
k_{\mathrm{nf}} & =k_{f}\left(1+4.4\left(\operatorname{Re}_{B}\right)^{0.4} \operatorname{Pr}^{0.66}\left(\frac{T}{T_{\mathrm{rf}}}\right)^{10}\left(\frac{k_{p}}{k_{f}}\right)^{0.03} \varphi^{0.66}\right), \\
\operatorname{Re}_{B} & =\frac{\rho_{f} u_{B} d_{p}}{\mu_{f}}, \\
u_{B} & =\frac{2 K_{B} T}{\left(\pi \mu_{f} d_{p}^{2}\right)},
\end{aligned}
$$

where $u_{B}$ is Brownian velocity, $T_{\mathrm{rf}}$ is a freezing point of a base fluid, and $K_{B}=1.380648 \times 10^{-23} \mathrm{~J} / \mathrm{K}$ is Boltzmann's coefficient.
2.1. Dimensionless Boundary Conditions. The dimensionless boundary conditions are

At a left wall of an outer square shape, $0 \leq Y \leq 2 L ; \theta=1, \Phi=1, U=V=0$,

At a right wall of an outer square shape, $0 \leq Y \leq 2 L ; \theta=0, \Phi=0, U=V=0$,

At top/bottom walls of an outer square shape, $0 \leq X \leq 2 L ; \frac{\partial \theta}{\partial Y}=0, \frac{\partial \Phi}{\partial Y}=0, U=V=0$,

At the walls of an inner cavity, $\frac{\partial \theta}{\partial n}=0, \frac{\partial \Phi}{\partial n}=0, U=V=0$, 
where $\mathbf{n}$ is a normal vector.

Predicted velocities:

\section{ISPH Method}

This section summarizes the following steps of the ISPH method.

$$
\begin{aligned}
U^{*}= & U^{n}+\varepsilon \frac{\rho_{f}}{\rho_{\mathrm{nf}}} \Delta \tau\left(\frac{1}{\varepsilon} \frac{\partial}{\partial X}\left(\operatorname{Pr} \frac{\mu_{\mathrm{nf}}}{\mu_{f}} \frac{\partial U}{\partial X}\right)+\frac{1}{\varepsilon} \frac{\partial}{\partial Y}\left(\operatorname{Pr} \frac{\mu_{\mathrm{nf}}}{\mu_{f}} \frac{\partial U}{\partial Y}\right)-\delta \frac{\operatorname{Pr} \mu_{\mathrm{eff}}}{\mu_{\mathrm{nf}} K^{*}(X, Y) \mathrm{Da}} U\right)^{n}, \\
V^{*}= & V^{n}+\varepsilon \frac{\rho_{f}}{\rho_{\mathrm{nf}}} \Delta \tau \\
& \cdot\left(\frac{1}{\varepsilon} \frac{\partial}{\partial X}\left(\operatorname{Pr} \frac{\mu_{\mathrm{nf}}}{\mu_{f}} \frac{\partial V}{\partial X}\right)+\frac{1}{\varepsilon} \frac{\partial}{\partial Y}\left(\operatorname{Pr} \frac{\mu_{\mathrm{nf}}}{\mu_{f}} \frac{\partial V}{\partial Y}\right)-\delta \frac{\operatorname{Pr} \mu_{\mathrm{eff}}}{\mu_{\mathrm{nf}} K^{*}(X, Y) \mathrm{Da}} V+\frac{(\rho \beta)_{\mathrm{nf}}}{(\rho \beta)_{f}} \operatorname{PrRa}(\theta+N \Phi)\right)^{n} .
\end{aligned}
$$

Pressure Poisson equation (PPE):

$$
\nabla^{2} P^{n+1}=\frac{\rho}{\Delta \tau}\left(\frac{\partial U^{*}}{\partial X}+\frac{\partial V^{*}}{\partial Y}\right)+\gamma \frac{\rho-\rho^{\text {num }}}{\Delta \tau^{2}} .
$$

Corrected velocities:

$$
\begin{aligned}
& U^{n+1}=U^{*}-\frac{\Delta \tau}{\rho}\left(\nabla P^{n+1}\right), \\
& V^{n+1}=V^{*}-\frac{\Delta \tau}{\rho}\left(\nabla P^{n+1}\right) .
\end{aligned}
$$

Thermal energy:

$$
\theta^{n+1}=\theta^{n}+\Delta \tau \frac{\left(\rho c_{p}\right)_{f}}{\left(\rho c_{p}\right)_{\mathrm{nf}}}\left[\frac{\partial}{\partial X}\left(\zeta(X, Y) \frac{\partial \theta}{\partial X}\right)+\frac{\partial}{\partial Y}\left(\zeta(X, Y) \frac{\partial \theta}{\partial Y}\right)\right]^{n} .
$$

Concentration equation:

$$
\Phi^{n+1}=\Phi^{n}+\Delta \tau \frac{1}{\operatorname{Le}}\left[\frac{\partial}{\partial X}\left(\Pi(X, Y) \frac{\partial \Phi}{\partial X}\right)+\frac{\partial}{\partial Y}\left(\Pi(X, Y) \frac{\partial \Phi}{\partial Y}\right)\right]^{n} .
$$

Positions are updated as

$$
\begin{aligned}
& X^{n+1}=X^{n}+\Delta \tau U^{n+1}, \\
& Y^{n+1}=Y^{n}+\Delta \tau V^{n+1} .
\end{aligned}
$$

Shifting technique is

$$
\Upsilon_{i^{\prime}}=\Upsilon_{i}+(\nabla \Upsilon)_{i} \delta R_{i i^{\prime}}+o\left(\delta R_{i i^{\prime}}^{2}\right) .
$$

3.1. SPH Formulation. Any hydrodynamic function $\Omega$ can be defined as

$$
\Omega\left(x_{i}, t\right)=\int W\left(r_{i j}, h\right) \Omega\left(x_{j}, t\right) \mathrm{d} v,
$$

where $h$ is a smoothing length and $W$ is a kernel function. Equation (13) is defined by SPH approximation as

$$
\Omega\left(x_{i}, t\right)=\sum_{j} \frac{m_{j}}{\rho_{j}} W\left(r_{i j}, h\right) \Omega_{j}\left(x_{j}, t\right) .
$$

Quintic spline kernel function is

$$
W\left(r_{i j}, h\right)=\alpha_{d} \begin{cases}\left(3-\frac{r_{i j}}{h}\right)^{5}-6\left(2-\frac{r_{i j}}{h}\right)^{5}+15\left(1-\frac{r_{i j}}{h}\right)^{5}, & 0 \leq r_{i j}<h, \\ \left(3-\frac{r_{i j}}{h}\right)^{5}-6\left(2-\frac{r_{i j}}{h}\right)^{5}, & h \leq r_{i j}<2 h, \\ \left(3-\frac{r_{i j}}{h}\right)^{5}, & 2 h \leq r_{i j}<3 h, \\ 0, & r_{i j} \geq 3 h,\end{cases}
$$


where $\alpha_{d}=7 / 478 \pi h^{2}$. First derivative is approximated by $\mathrm{SPH}$ :

$$
\begin{aligned}
& \nabla \Omega\left(r_{i}\right)=\frac{1}{\rho_{i}} \sum_{j} m_{j}\left(\Omega_{j}-\Omega_{i}\right) \nabla W\left(r_{i j}, h\right), \\
& \nabla \phi\left(r_{i}\right)=\rho_{i} \sum_{j} m_{j}\left(\frac{\Omega_{j}}{\rho_{j}^{2}}+\frac{\Omega_{i}}{\rho_{i}^{2}}\right) \nabla W\left(r_{i j}, h\right) .
\end{aligned}
$$

First derivatives of velocity $\nabla \mathbf{u}_{i}$ and pressure $\nabla P_{i}$ are defined as

$$
\begin{aligned}
& \nabla \mathbf{u}_{i}=\frac{1}{\rho_{i}} \sum_{j} m_{j}\left(\mathbf{u}_{j}-\mathbf{u}_{i}\right) \cdot \nabla W\left(r_{i j}, h\right), \\
& \nabla P_{i}=\rho_{i} \sum_{j} m_{j}\left(\frac{P_{j}}{\rho_{j}^{2}}+\frac{P_{i}}{\rho_{i}^{2}}\right) \nabla W\left(r_{i j}, h\right) .
\end{aligned}
$$

Here, first derivative of a velocity is corrected by a kernel gradient normalization $\widehat{L}\left(r_{i j}\right)$ as

$$
\widehat{\nabla} W_{i j}=\widehat{L}\left(r_{i j}\right) \nabla W_{i j}, \widehat{L}\left(r_{i j}\right)=\left(\begin{array}{l}
\sum_{j=1}^{n} \frac{m_{j}}{\rho_{j}}\left(x_{j}-x_{i}\right) \frac{\partial W_{i j}}{\partial x_{i}} \sum_{j=1}^{n} \frac{m_{j}}{\rho_{j}}\left(x_{j}-x_{i}\right) \frac{\partial W_{i j}}{\partial y_{i}} \\
\left.\sum_{j=1}^{n} \frac{m_{j}}{\rho_{j}}\left(y_{j}-y_{i}\right) \frac{\partial W_{i j}}{\partial x_{i}} \sum_{j=1}^{n} \frac{m_{j}}{\rho_{j}}\left(y_{j}-y_{i}\right) \frac{\partial W_{i j}}{\partial y_{i}}\right)^{-1} .
\end{array}\right.
$$

SPH approximations of second derivatives for the velocity and pressure are

$$
\begin{aligned}
\nabla \cdot(\nu \nabla \cdot \mathbf{u}) & =\sum_{j} m_{j}\left(\frac{\rho_{i} \nu_{i}+\rho_{j} \nu_{j}}{\rho_{i} \rho_{j}} \frac{\mathbf{r}_{i j} \cdot \nabla W_{i j}}{r_{i j}^{2}+\eta^{2}}\right) \mathbf{u}_{i j}, \\
\nabla^{2} P_{i} & =\frac{2}{\rho_{i}} \sum_{j} m_{j}\left(\frac{\mathbf{r}_{i j} \cdot \nabla W_{i j}}{r_{i j}^{2}+\eta^{2}}\right) P_{i j} .
\end{aligned}
$$

The calculations of FORTRAN-90 code of the ISPH method were performed by Shaheen II supercomputer owned by King Abdullah University of Science and Technology, Thuwal, Saudi Arabia (project number K1467) (https://www.hpc.kaust.edu.sa/content/shaheen-ii).

\section{Validation Test}

In this section, the validation test of the ISPH method for the problem of natural convection in a partitioned porous cavity has been conducted. Figure 2 shows the temperature profiles in a partitioned porous cavity among the results from the current results of the ISPH method and Beckermann et al. [48]. The temperature profiles were evaluated at three different locations in a cavity: $Y / L=0.055, Y / L=0.496$, and $Y / L=0.874$. It is clear that the results of the ISPH method have a well agreement with the experimental and numerical results of Beckermann et al. [48].

\section{Results and Discussion}

The numerical results are presented in terms of concentration, temperature, and velocity field characteristics in order to get a clear insight on the problem of doublediffusive of a nanofluid in an outer square shape mounted on the four vertical gates in a porous cavity. Figures 3-5 show the impacts of a buoyancy ratio parameter on the characteristic of the temperature, concentration, and velocity field. It is revealed that an increase in a buoyancy ratio parameter rises the temperature and concentration distributions inside an outer square shape and inner square cavity. Moreover, at opposing flow mode $(N<0)$, the double-diffusive convection occurs in the bottom area of an outer square shape and in an inner cavity. While at aiding flow mode $(N>0)$, the double-diffusive convection occurs in the top area of an outer square shape and in a cavity. The maximum of the velocity field increases by $175.75 \%$ as the buoyancy ratio parameter increases from $N=-2$ to $N=2$. Moreover, the maximum of the velocity field appears in the left-top area of an outer square shape at opposing flow mode $(N<0)$, and it appears in the bottom-left part of an outer square shape at aiding flow mode $(N>0)$.

The effects of the Darcy parameter on the characteristic of the temperature, concentration, and velocity field have been shown in Figures 6-8. As a Darcy parameter represents a useful factor for explaining the fluid flow in the porous materials, a little decrease in the temperature and concentration distributions is occurring when the Darcy parameter decreases from $10^{-3}$ to $10^{-5}$. Maximum of the velocity field is reducing as a Darcy parameter decreases from $10^{-3}$ to $10^{-5}$. Moreover, a lower velocity field in the center of a square cavity appears at a lower Darcy parameter $\mathrm{Da} \leq 10^{-4}$. From these figures, it is noted there are slight changes in the characteristics of the temperature, concentration, and velocity field below the effects of a Darcy parameter. The physical reason returns to the presence of a porous medium layer in the inner cavity only, while an outer square shape contains nanofluid only.

Figures 9-11 introduce the influences of the solid volume fraction on the characteristics of temperature, concentration, and velocity field. It is seen that the temperature and concentration distributions have slight decrease according 


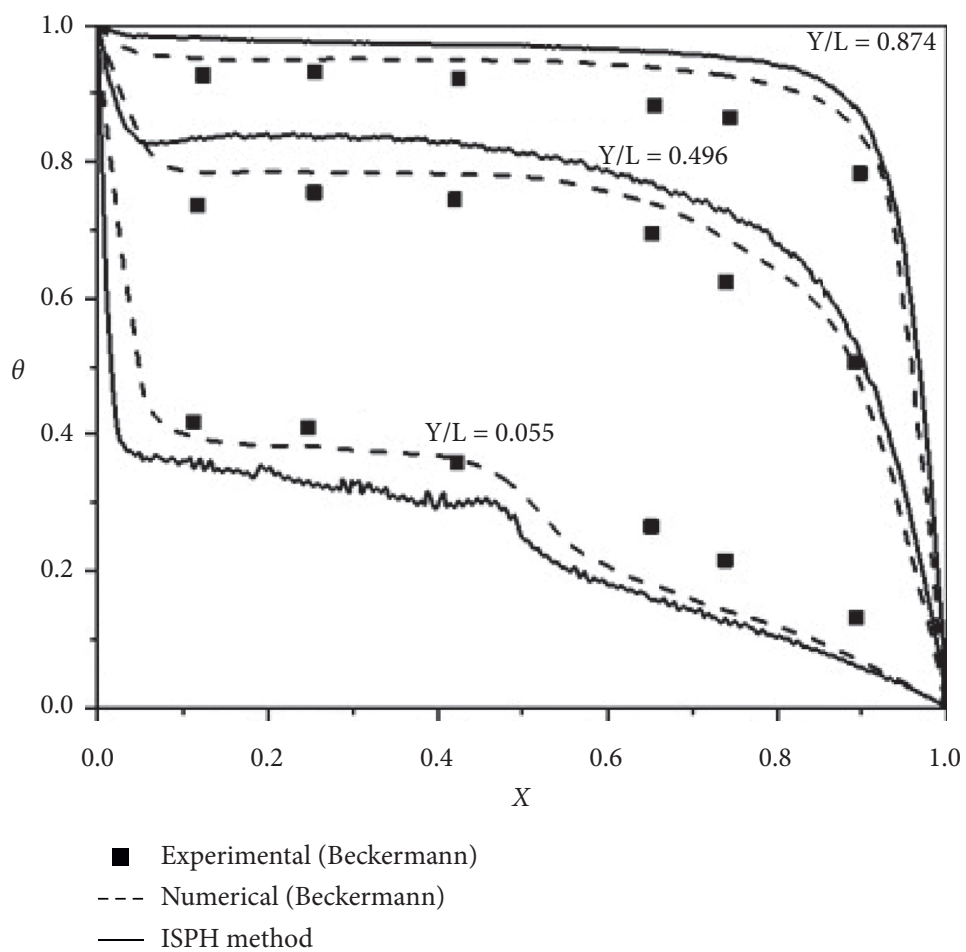

FIGURE 2: Comparison of the temperature profiles in a partitioned porous cavity between Beckermann et al. [48] and ISPH method.

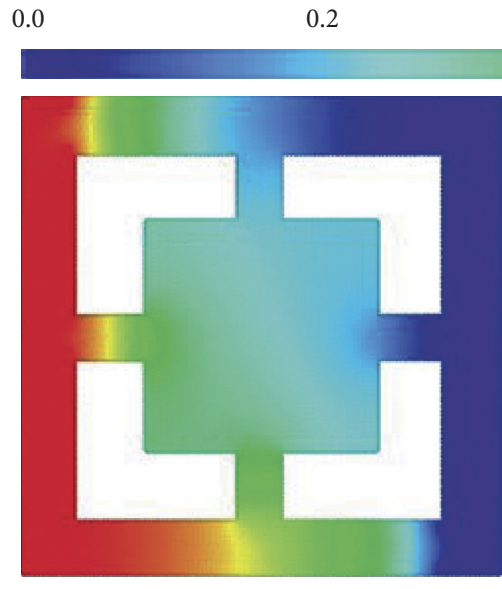
0.4 0.6 0.8 1.0

$$
N=-2
$$

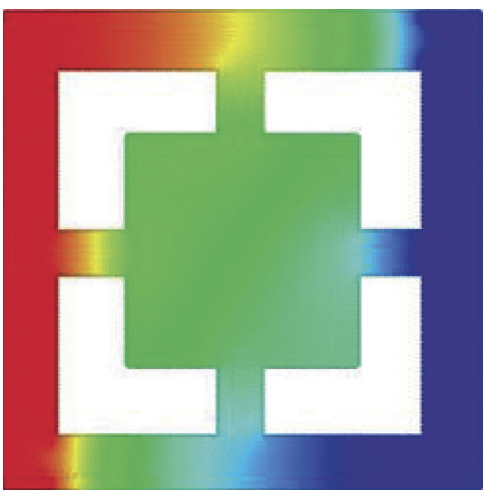

$N=0$

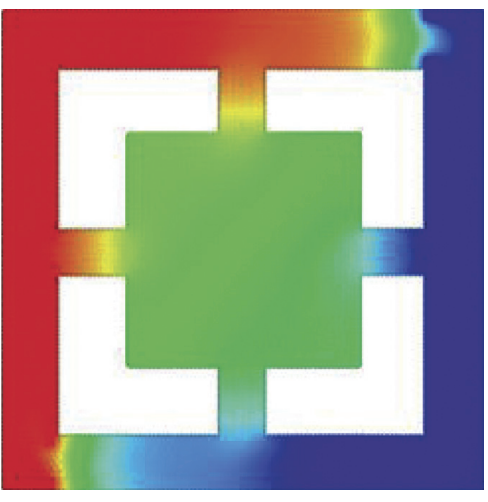

$N=2$

FIGURE 3: Features of temperature below the variations of a buoyancy ratio parameter at $\mathrm{Ra}=10^{4}, \phi=0.01, \mathrm{Da}=10^{-3}, \eta_{1}=\eta_{2}=1.5$, $\varepsilon=0.6$, Le $=20$, and $\tau=1$.

to adding more concentration of nanoparticles until 5\%. The concentration of the nanoparticles is limited to $5 \%$ to avoid solidification between the nanofluid and porous media. Adding more concentration of the nanoparticles increases the nanofluid viscosity. As a result, the maximum of the velocity field decreases by $44.77 \%$ as the solid volume fraction increases from 0 to 0.05 .

The variations of the porous levels according to different $\eta_{1}$ and $\eta_{2}$ on the temperature, concentration, and velocity field profiles have been shown in Figures 12-14. In Figure 12, the highest distributions of the temperature are obtained at a heterogeneous porous medium $\eta_{1}=\eta_{2}=1.5$ and lowest temperature distributions are obtained at a homogeneous porous medium $\eta_{1}=\eta_{2}=0$. In Figure 13, it is seen that the concentration distributions are affected strongly by changing the porous medium levels. A heterogeneous porous medium $\eta_{1}=\eta_{2}=1.5$ gives the highest concentration distributions in an inner cavity and lowest concentration distributions in an outer square shape. In contrast, a homogeneous porous medium $\eta_{1}=\eta_{2}=0$ gives the lowest concentration distributions in an inner cavity and the highest concentration distributions in an outer square shape. In Figure 12, there are slight changes in the velocity field below different levels of porous media. The physical reason 
0.0

0.2

0.4

0.6

0.8

1.0

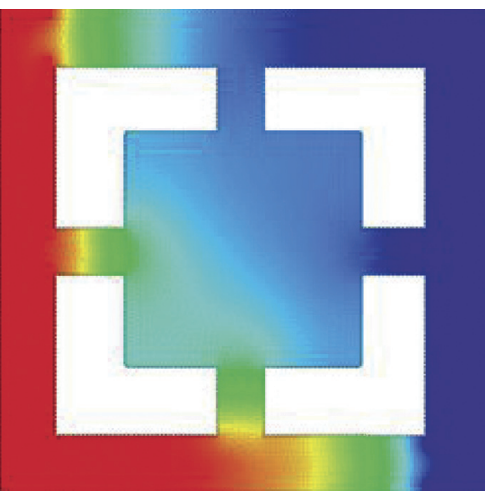

$N=-2$

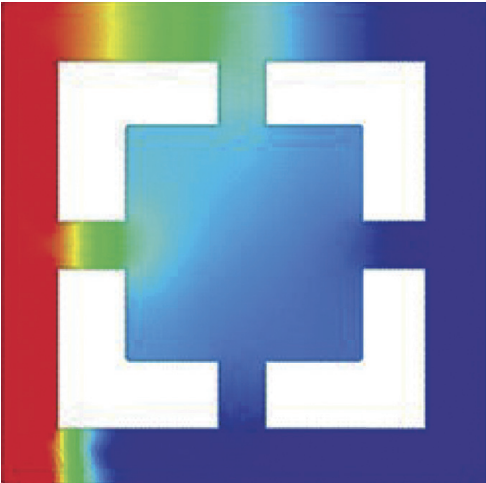

$N=0$

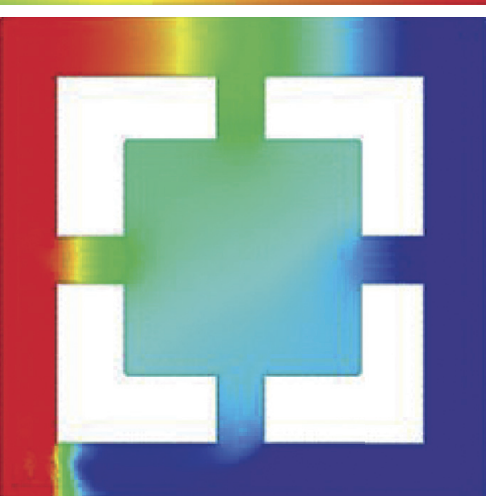

$N=2$

FIgURE 4: Features of concentration below the variations in a buoyancy ratio parameter at $\operatorname{Ra}=10^{4}, \phi=0.01, \mathrm{Da}=10^{-3}, \eta_{1}=\eta_{2}=1.5$, $\varepsilon=0.6, \mathrm{Le}=20$, and $\tau=1$.
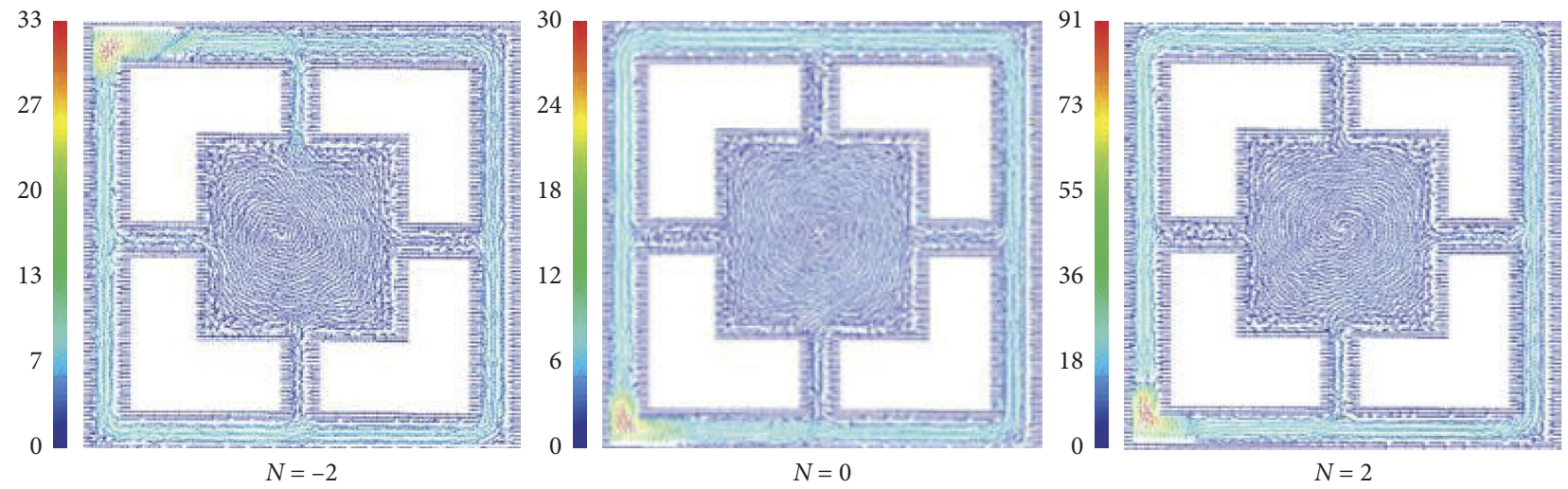

FIGURE 5: Velocity field below the variations of a buoyancy ratio parameter at $\mathrm{Ra}=10^{4}, \phi=0.01, \mathrm{Da}=10^{-3}, \eta_{1}=\eta_{2}=1.5, \varepsilon=0.6, \mathrm{Le}=20$, and $\tau=1$.

0.0

0.2

0.4

0.6

0.8

1.0

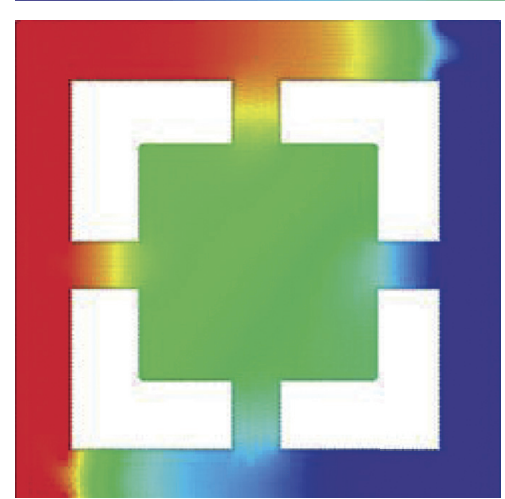

$\mathrm{Da}=10^{-3}$

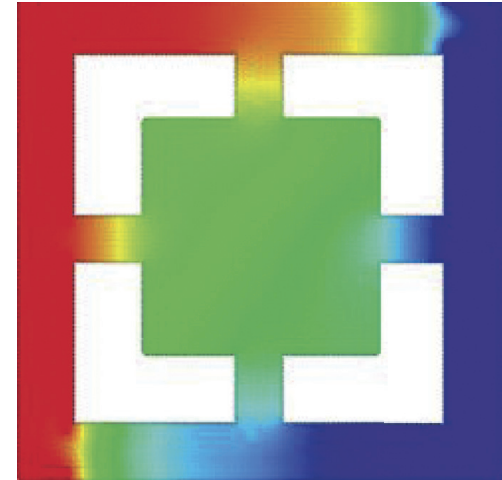

$\mathrm{Da}=10^{-4}$

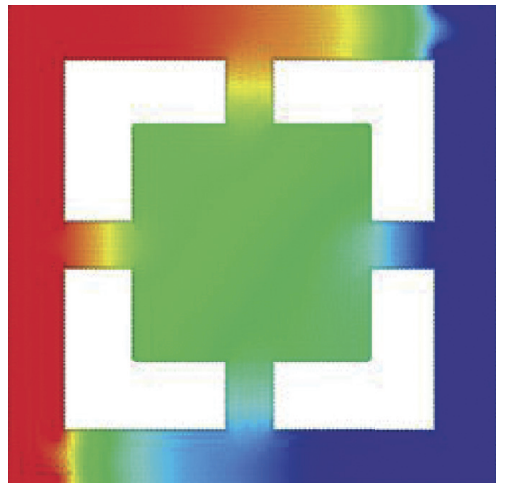

$D a=10^{-5}$

FIGURE 6: Features of temperature below the variations of a Darcy parameter at $N=1, \mathrm{Ra}=10^{4}, \phi=0.01, \eta_{1}=\eta_{2}=1.5, \varepsilon=0.6, \mathrm{Le}=20$, and $\tau=1$. 


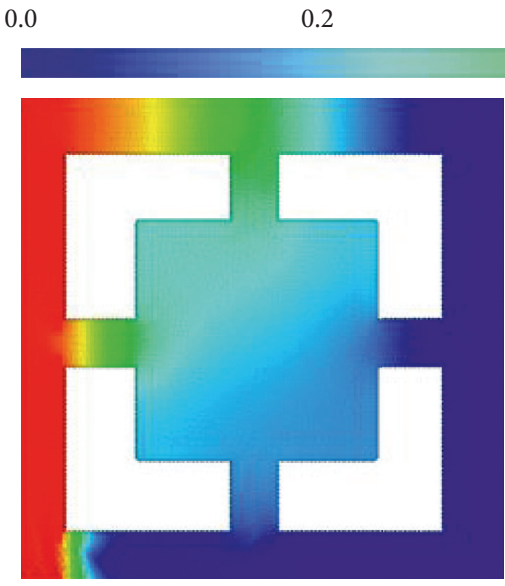

$D a=10^{-3}$
0.4

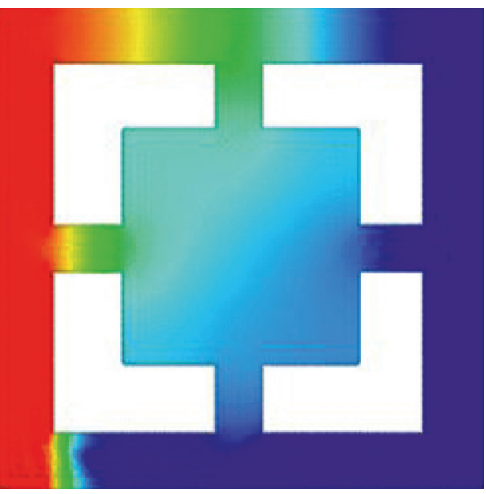

$D a=10^{-4}$

0.6

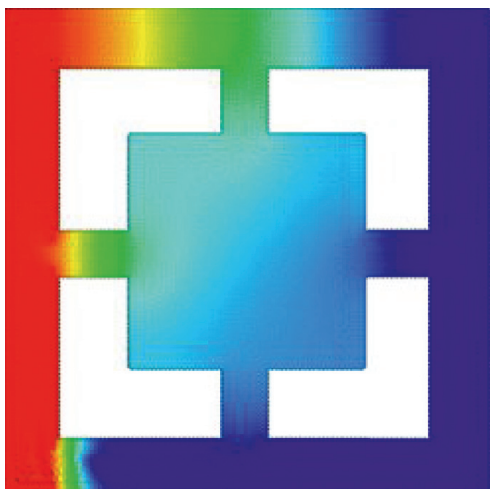

$D a=10^{-5}$

FIGURE 7: Features of concentration below the variations of a Darcy parameter at $N=1, \mathrm{Ra}=10^{4}, \phi=0.01, \eta_{1}=\eta_{2}=1.5, \varepsilon=0.6, \mathrm{Le}=20$, and $\tau=1$.

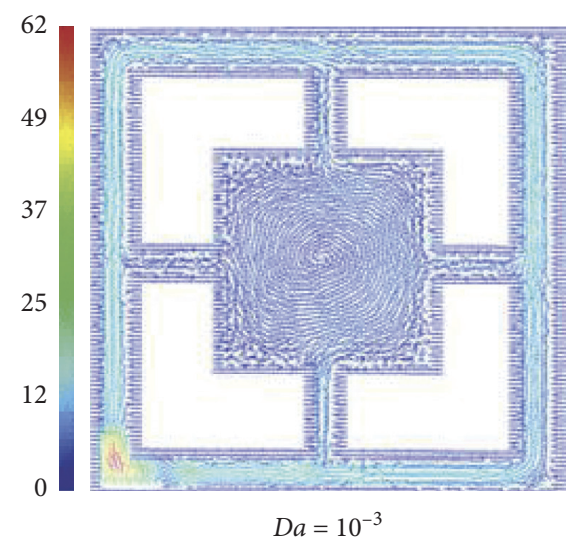

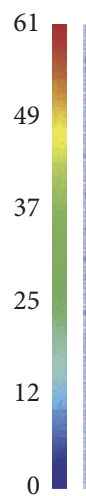

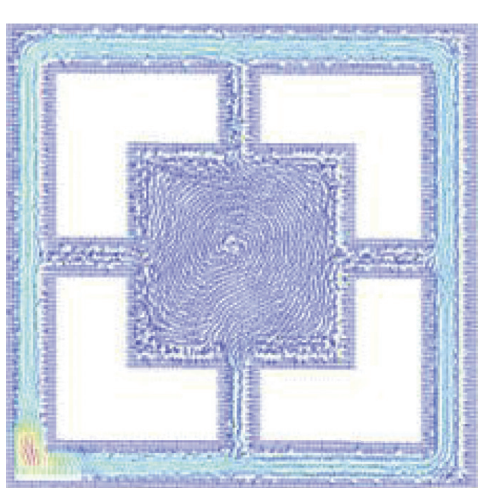

$D a=10^{-4}$

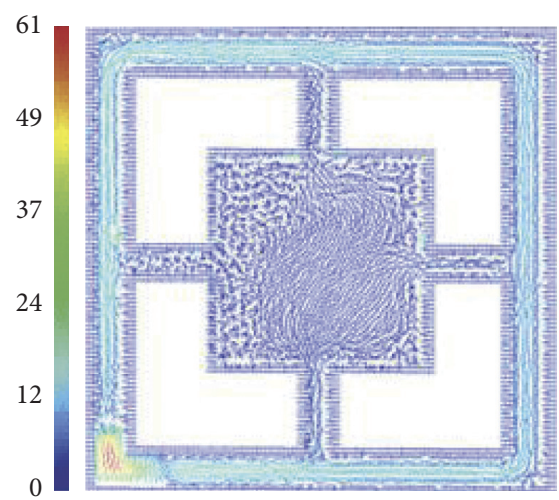

$D a=10^{-5}$

Figure 8: Velocity field below the variations of a Darcy parameter at $N=1, \mathrm{Ra}=10^{4}, \phi=0.01, \eta_{1}=\eta_{2}=1.5, \varepsilon=0.6, \mathrm{Le}=20$, and $\tau=1$.

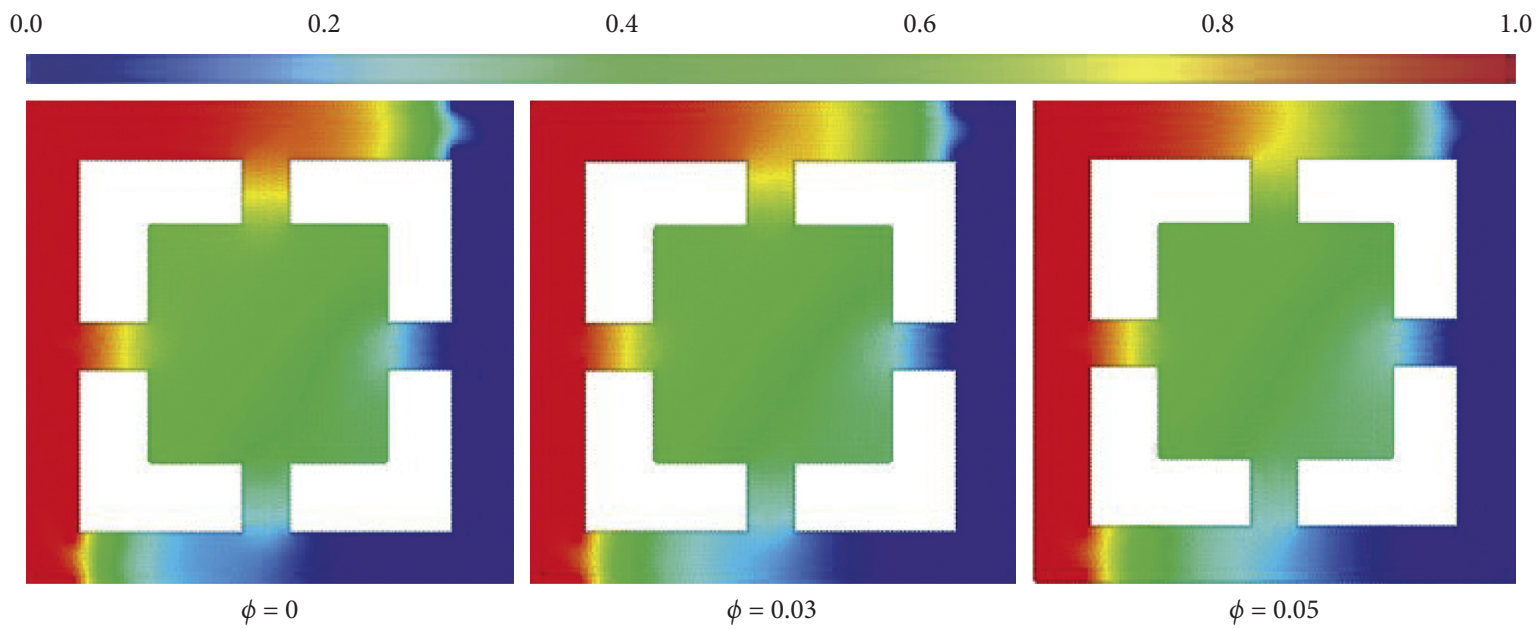

FIGURE 9: Features of temperature below variations of a solid volume fraction parameter at $N=1, \mathrm{Ra}=10^{4}, \mathrm{Da}=10^{-3}, \eta_{1}=\eta_{2}=1.5$, $\varepsilon=0.6$, Le $=20$, and $\tau=1$. 
0.0

0.2

0.4

0.6

0.8

1.0

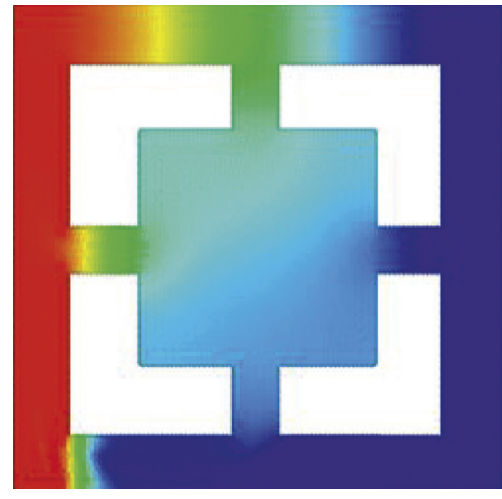

$\phi=0$

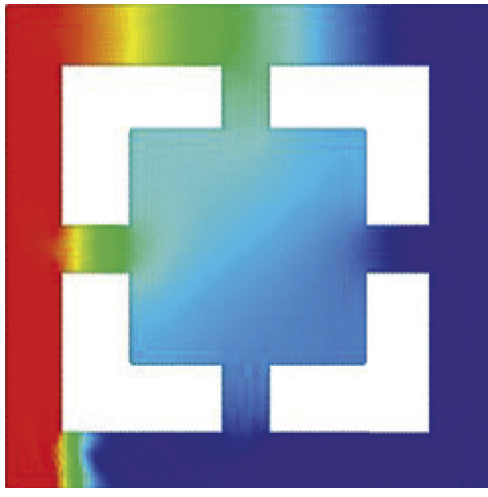

$\phi=0.03$

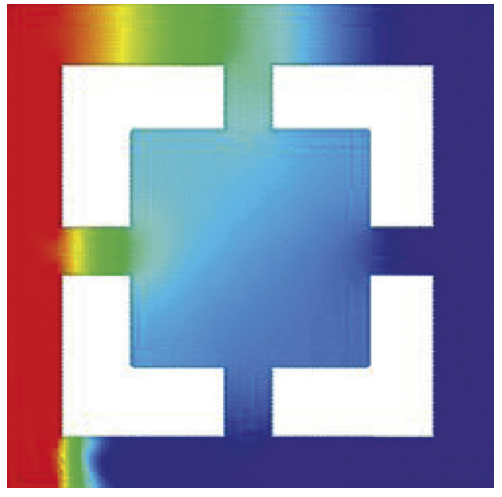

$\phi=0.05$

FIGURE 10: Features of concentration below the variations of a solid volume fraction parameter at $N=1, \mathrm{Ra}=10^{4}, \mathrm{Da}=10^{-3}, \eta_{1}=\eta_{2}=1.5$, $\varepsilon=0.6, \mathrm{Le}=20$, and $\tau=1$.
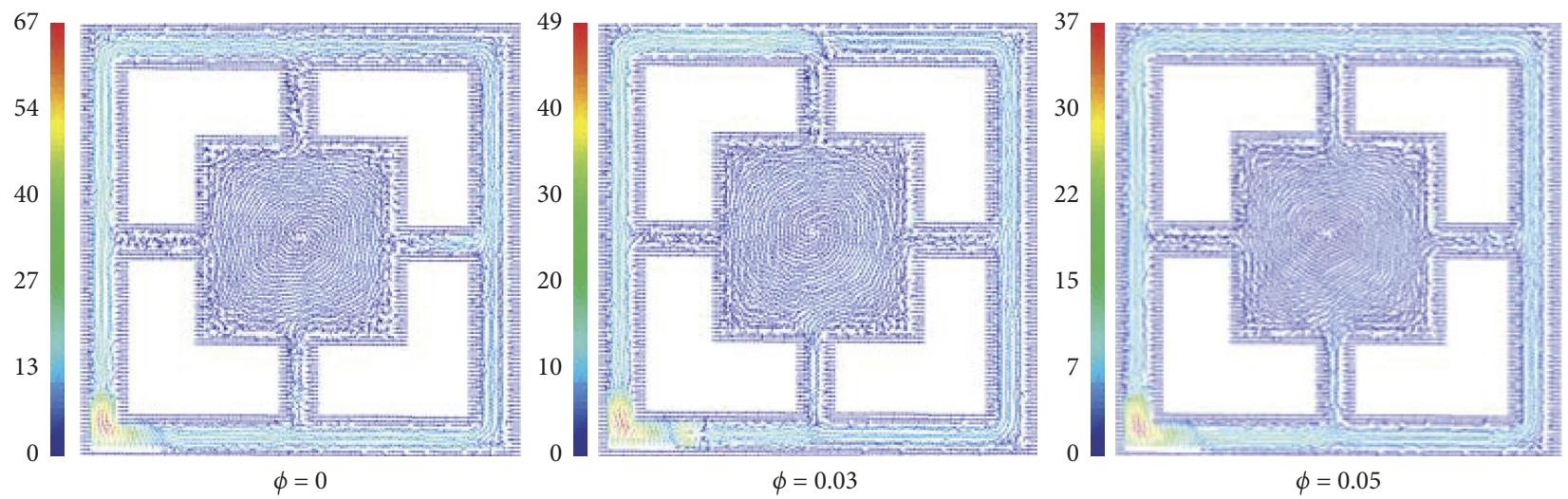

Figure 11: Velocity field below the variations of a solid volume fraction parameter at $N=1, \operatorname{Ra}=10^{4}, \mathrm{Da}=10^{-3}, \eta_{1}=\eta_{2}=1.5$, $\varepsilon=0.6, \mathrm{Le}=20$, and $\tau=1$.

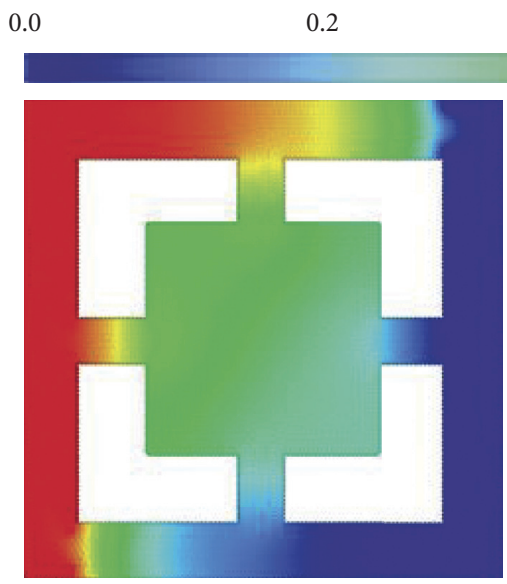

$\eta_{1}=\eta_{2}=0$
0.4

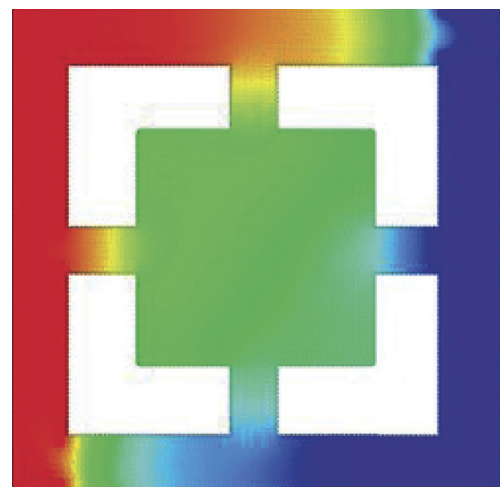

$\eta_{1}=\eta_{2}=0.5$

0.6

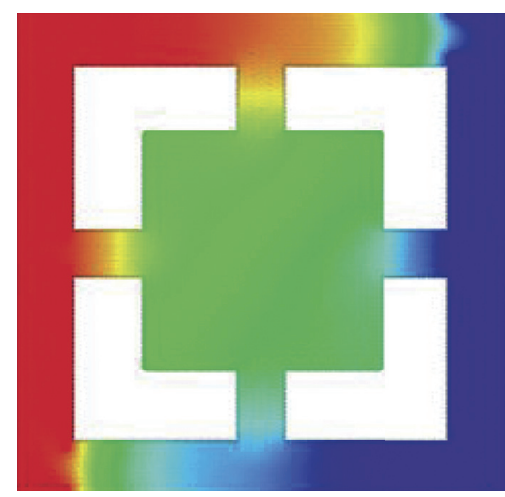

$\eta_{1}=\eta_{2}=1.5$

FIGURE 12: Features of temperature below the variations of a porous level, $\eta_{1}$ and $\eta_{2}$, at $N=1, \phi=0.01, \mathrm{Ra}=10^{4}, \mathrm{Da}=10^{-4}, \varepsilon=0.6$, Le $=20$, and $\tau=1$. 


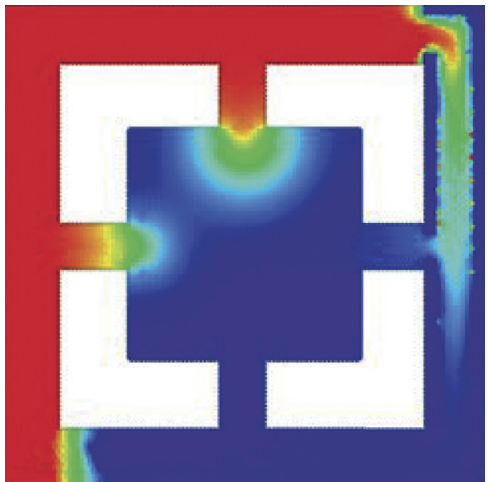

$\eta_{1}=\eta_{2}=0$

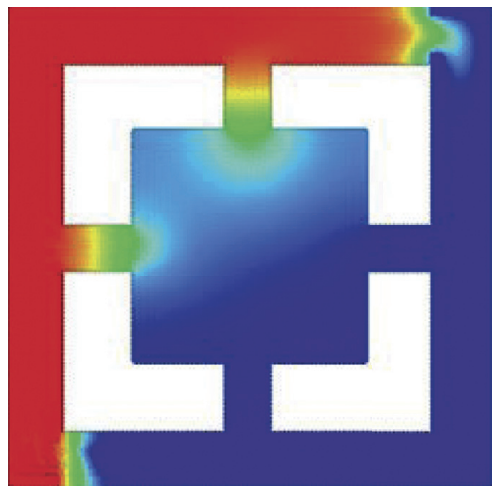

$\eta_{1}=\eta_{2}=0.5$

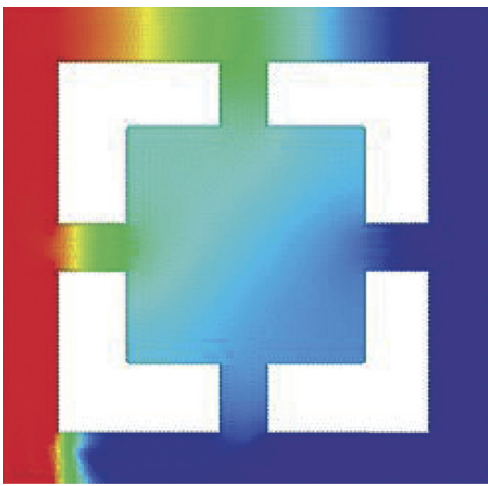

$\eta_{1}=\eta_{2}=1.5$

FiguRE 13: Features of concentration below the variations of a porous level, $\eta_{1}$ and $\eta_{2}$, at $N=1, \phi=0.01, \mathrm{Ra}=10^{4}, \mathrm{Da}=10^{-4}, \varepsilon=0.6$, $\mathrm{Le}=20$, and $\tau=1$.
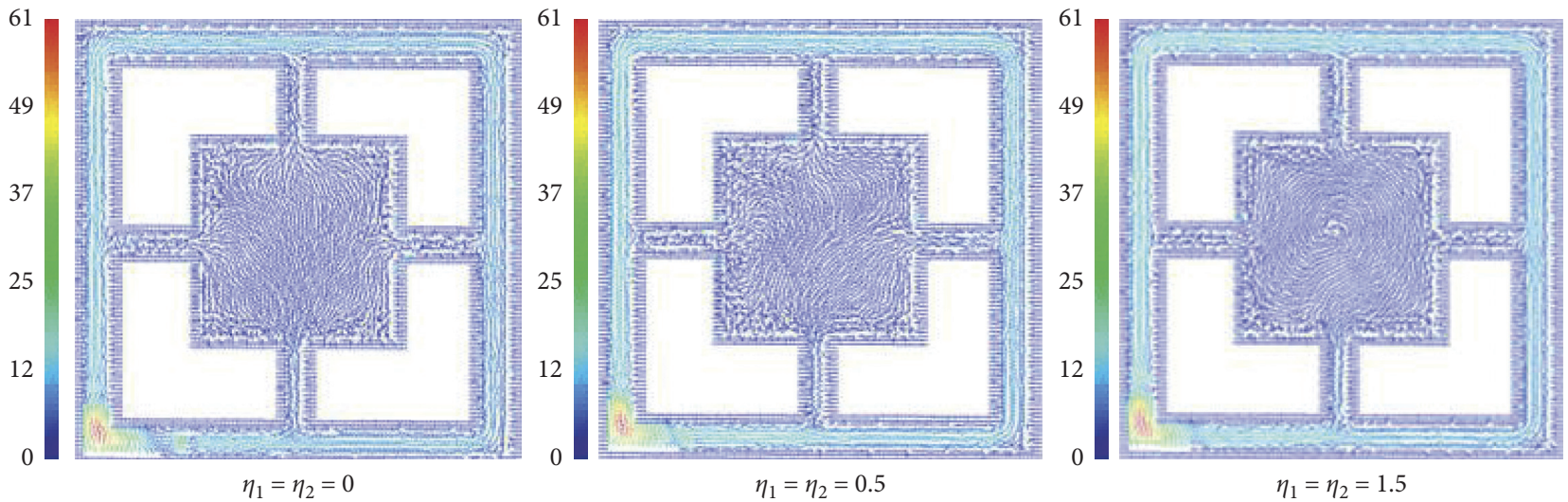

Figure 14: Features of concentration below the variations of a porous level, $\eta_{1}$ and $\eta_{2}$, at $N=1, \phi=0.01, \mathrm{Ra}=10^{4}, \mathrm{Da}=10^{-4}, \varepsilon=0.6$, Le $=20$, and $\tau=1$.

returns to the presence of porous media in an inner cavity only.

\section{Conclusion}

The novelty of this work is to simulate double-diffusive convection of nanofluid inside a new novel shape of two square cavities connected by four vertical gates. Three different levels of porous media related to the values of $\eta_{1}$ and $\eta_{2}$ were conducted. An inner cavity is filled by a heterogeneous porous medium and $\mathrm{Al}_{2} \mathrm{O}_{3}$-water nanofluid. The outer square shape with four gates is filled by a nanofluid only. An incompressible version of the SPH method was utilized to solve the nondimensional governing equations of the current problem. The main finding of the current results can be summarized as follows: (i) An augmentation in a buoyancy ratio parameter increases the temperature and concentration distributions in an outer square shape and in an inner cavity

(ii) At opposing flow mode, $N<0$, and the direction of buoyancy force from upwards to downwards and the maximum of velocity field occur at a top-left part of an outer square shape

(iii) At aiding flow mode, $N>0$, and the direction of buoyancy force from downwards into upwards and the maximum of velocity field occur at a bottom-left part of an outer square shape

(iv) A decrease in the Darcy parameter reduces the flow speed in an inner porous cavity, and it has slight effects in an outer square shape 
(v) Adding more concentration of nanoparticles increases the viscosity of a host fluid, and consequently, the flow speed inside an outer square shape and in an inner cavity decreases

(vi) Variation in porous medium levels changes the concentration distributions inside an outer square shape and in an inner cavity

\section{Nomenclature}

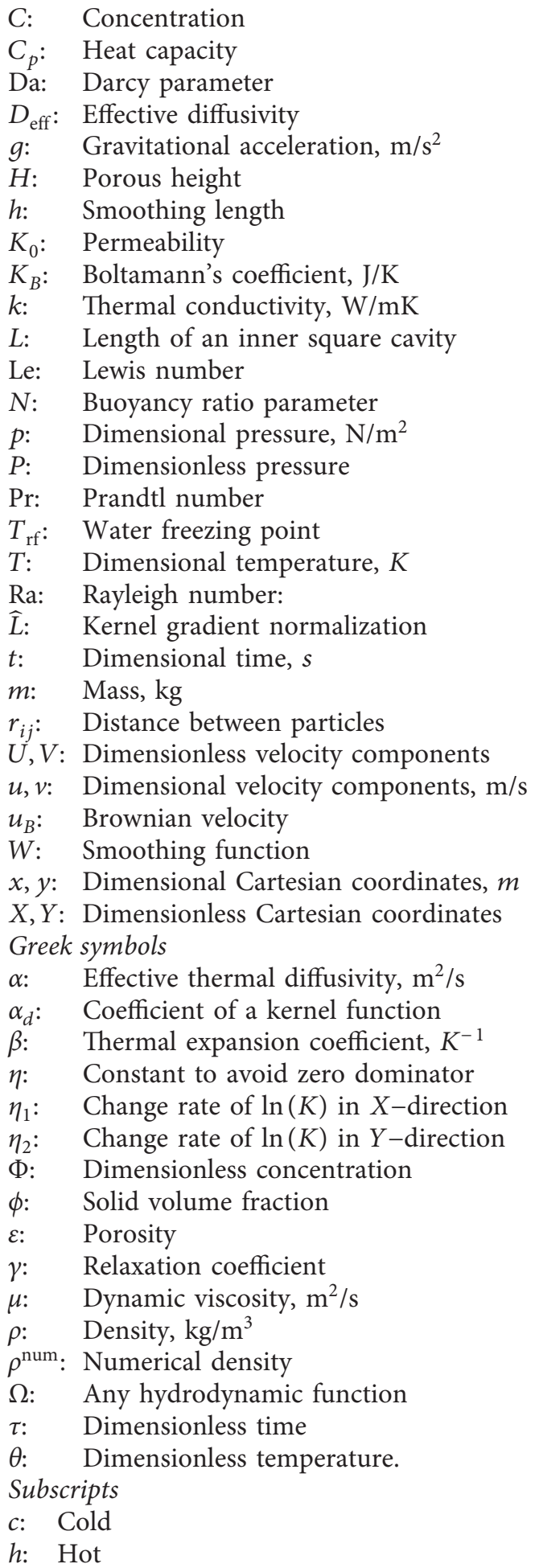

$f$ : Fluid

nf: Nanofluid

$p$ : Porous medium.

\section{Data Availability}

Data used to support the findings of the study are available from the corresponding author upon request.

\section{Conflicts of Interest}

The authors declare that there are no conflicts of interest associated with this publication.

\section{Acknowledgments}

The first author extends his appreciation to the Deanship of Scientific Research at King Khalid University, Abha, Saudi Arabia, for funding this work through the Research Group Project under Grant no. RGP.2/17/42. This work was supported by the National Natural Science Foundation of China (no. 71601072), Key Scientific Research Project of Higher Education Institutions in Henan Province of China (no. 20B110006), and the Fundamental Research Funds for the Universities of Henan Province.

\section{References}

[1] H. E. Huppert and J. S. Turner, "Double-diffusive convection," Journal of Fluid Mechanics, vol. 106, no. -1, pp. 299-329, 1981.

[2] B. T. Murray and C. F. Chen, "Double-diffusive convection in a porous medium," Journal of Fluid Mechanics, vol. 201, no. -1 , pp. 147-166, 1989.

[3] M. Sankar, B. Kim, and J. M. Lopez, "Younghae Do, Thermosolutal convection from a discrete heat and solute source in a vertical porous annulus," International Journal of Heat and Mass Transfer, vol. 55, no. 15-16, pp. 4116-4128, 2012.

[4] H. Beji, R. Bennacer, R. Duval, and P. Vasseur, "Doublediffusive natural convection in a vertical porous annulus," Numerical Heat Transfer, Part A: Applications, vol. 36, no. 2, pp. 153-170, 1999.

[5] M. Marcoux, M.-C. Charrier-Mojtabi, and M. Azaiez, "Double-diffusive convection in an annular verticalporous layer," International Journal of Heat and Mass Transfer, vol. 42, no. 13, pp. 2313-2325, 1999.

[6] P. Nithiarasu, K. N. Seetharamu, and T. Sundararajan, "NonDarcy double-diffusive natural convection in axisymmetric fluid saturated porous cavities," Heat and Mass Transfer, vol. 32, no. 6, pp. 427-433, 1997.

[7] J. Lee, S. H. Kang, and S. Young, "Numerical study of multilayered flow regime in double-diffusive convection in a rotating annulus with lateral heating," Numerical Heat Transfer, Part A: Applications, vol. 38, no. 5, pp. 467-489, 2000.

[8] S. Chen, J. Tölke, and M. Krafczyk, "Numerical investigation of double-diffusive (natural) convection in vertical annuluses with opposing temperature and concentration gradients," International Journal of Heat and Fluid Flow, vol. 31, no. 2, pp. 217-226, 2010.

[9] B. Goyeau, J.-P. Songbe, and D. Gobin, "Numerical study of double-diffusive natural convection in a porous cavity using 
the Darcy-Brinkman formulation," International Journal of Heat and Mass Transfer, vol. 39, no. 7, pp. 1363-1378, 1996.

[10] A. J. Chamkha and A. Al-Mudhaf, "Double-diffusive natural convection in inclined porous cavities with various aspect ratios and temperature-dependent heat source or sink," Heat and Mass Transfer, vol. 44, no. 6, p. 679, 2008.

[11] O. Mahian, A. Kianifar, S. A. Kalogirou, I. Pop, and S. Wongwises, "A review of the applications of nanofluids in solar energy," International Journal of Heat and Mass Transfer, vol. 57, no. 2, pp. 582-594, 2013.

[12] Y. Wang, Q. Liu, J. Lei, and H. Jin, "Performance analysis of a parabolic trough solar collector with non-uniform solar flux conditions," International Journal of Heat and Mass Transfer, vol. 82, pp. 236-249, 2015.

[13] M. J. Muhammad, I. A. Muhammad, N. A. C. Sidik, M. N. A. W. M. Yazid, R. Mamat, and G. Najafi, "Retracted: the use of nanofluids for enhancing the thermal performance of stationary solar collectors: a review," Renewable and Sustainable Energy Reviews, vol. 63, pp. 226-236, 2016.

[14] A. H. Elsheikh, S. W. Sharshir, M. E. Mostafa, F. A. Essa, and M. K. Ahmed Ali, "Applications of nanofluids in solar energy: a review of recent advances," Renewable and Sustainable Energy Reviews, vol. 82, pp. 3483-3502, 2018.

[15] K. Khanafer and K. Vafai, "A review on the applications of nanofluids in solar energy field," Renewable Energy, vol. 123, pp. 398-406, 2018.

[16] J. Buongiorno, L.-W. Hu, S. J. Kim, R. Hannink, B. Truong, and E. Forrest, "Nanofluids for enhanced economics and safety of nuclear reactors: an evaluation of the potential Features, issues, and research gaps," Nuclear Technology, vol. 162, no. 1, pp. 80-91, 2008.

[17] J. Buongiorno, L. W. Hu, G. Apostolakis, R. Hannink, T. Lucas, and A. Chupin, "A feasibility assessment of the use of nanofluids to enhance the in-vessel retention capability in light-water reactors," Nuclear Engineering and Design, vol. 239, no. 5, pp. 941-948, 2009.

[18] M. Molana, "A comprehensive review on the nanofluids application in the tubular heat exchangers," American Journal of Heat Mass Transfer: Advancement in Process Modelling, vol. 3, no. 5, pp. 352-381, 2016.

[19] G. Diglio, C. Roselli, M. Sasso, and U. Jawali Channabasappa, "Borehole heat exchanger with nanofluids as heat carrier," Geothermics, vol. 72, pp. 112-123, 2018.

[20] M. M. Bhatti, S. R. Mishra, T. Abbas, and M. M. Rashidi, "A mathematical model of MHD nanofluid flow having gyrotactic microorganisms with thermal radiation and chemical reaction effects," Neural Computing and Applications, vol. 30, no. 4, pp. 1237-1249, 2018.

[21] R. Mohebbi and M. M. Rashidi, "Numerical simulation of natural convection heat transfer of a nanofluid in an L-shaped enclosure with a heating obstacle," Journal of the Taiwan Institute of Chemical Engineers, vol. 72, pp. 70-84, 2017.

[22] R. Chowdhury, S. Parvin, and M. A. H. Khan, "Finite element analysis of double-diffusive natural convection in a porous triangular enclosure filled with $\mathrm{Al} 2 \mathrm{O} 3$-water nanofluid in presence of heat generation," Heliyon, vol. 2, Article ID e00140, 2016.

[23] A. M. Aly and Z. A. S. Raizah, "Double-diffusive natural convection in an enclosure filled with nanofluid using ISPH method," Alexandria Engineering Journal, vol. 55, no. 4, pp. 3037-3052, 2016.

[24] St. I. Gheorghitza, "The marginal stability in porous inhomogeneous media," in Mathematical Proceedings of the
Cambridge Philosophical Society, pp. 871-877, Cambridge University Press, Cambridge, EN, USA, 1961.

[25] R. McKibbin and M. J. O'Sullivan, "Onset of convection in a layered porous medium heated from below," Journal of Fluid Mechanics, vol. 96, no. 2, pp. 375-393, 1980.

[26] P. A. Domenico and F. W. Schwartz, Physical and Chemical Hydro-Geology, Wiley, New York, NY, USA, 1998.

[27] L. B. Lucy, "A numerical approach to the testing of the fission hypothesis," The Astronomical Journal, vol. 82, pp. 1013-1024, 1977.

[28] R. A. Gingold and J. J. Monaghan, "Smoothed particle hydrodynamics: theory and application to non-spherical stars," Monthly Notices of the Royal Astronomical Society, vol. 181, no. 3, pp. 375-389, 1977.

[29] J. J. Monaghan, "Simulating free surface flows with SPH," Journal of Computational Physics, vol. 110, no. 2, pp. 399-406, 1994.

[30] X. Sun, M. Sakai, and Y. Yamada, "Three-dimensional simulation of a solid-liquid flow by the DEM-SPH method," Journal of Computational Physics, vol. 248, pp. 147-176, 2013.

[31] K. Szewc, J. Pozorski, and A. Tanière, "Modeling of natural convection with smoothed particle hydrodynamics: nonBoussinesq formulation," International Journal of Heat and Mass Transfer, vol. 54, no. 23-24, pp. 4807-4816, 2011.

[32] N. Tofighi, M. Ozbulut, A. Rahmat, J. J. Feng, and M. Yildiz, "An incompressible smoothed particle hydrodynamics method for the motion of rigid bodies in fluids," Journal of Computational Physics, vol. 297, pp. 207-220, 2015.

[33] Z.-B. Wang, R. Chen, H. Wang, Q. Liao, X. Zhu, and S.-Z. Li, "An overview of smoothed particle hydrodynamics for simulating multiphase flow," Applied Mathematical Modelling, vol. 40, no. 23-24, pp. 9625-9655, 2016.

[34] M. Yildiz, R. A. Rook, and A. Suleman, "SPH with the multiple boundary tangent method," International Journal for $\mathrm{Nu}$ merical Methods in Engineering, vol. 77, no. 10, pp. 1416-1438, 2009.

[35] Z. L. Zhang, T. Long, J. Z. Chang, and M. B. Liu, "A smoothed particle element method (SPEM) for modeling fluid-structure interaction problems with large fluid deformations," Computer Methods in Applied Mechanics and Engineering, vol. 356, pp. 261-293, 2019.

[36] A. M. Aly and M. Asai, "Incompressible smoothed particle hydrodynamics simulations of fluid-structure interaction on free surface flows," International Journal of Fluid Mechanics Research, vol. 41, no. 6, pp. 471-484, 2014.

[37] A. Khayyer, H. Gotoh, and S. D. Shao, "Corrected Incompressible SPH method for accurate water-surface tracking in breaking waves," Coastal Engineering, vol. 55, no. 3, pp. 236-250, 2008.

[38] A. Khayyer and H. Gotoh, "A higher order Laplacian model for enhancement and stabilization of pressure calculation by the MPS method," Applied Ocean Research, vol. 32, no. 1, pp. 124-131, 2010.

[39] A. E. Abouelregal and H. Ahmad, "Thermodynamic modeling of viscoelastic thin rotating microbeam based on non-Fourier heat conduction," Applied Mathematical Modelling, vol. 91, 2020.

[40] A. M. Aly, "ISPH method for MHD convective flow from grooves inside a nanofluid-filled cavity under the effects of Soret and Dufour numbers," Physica A: Statistical Mechanics and Its Applications, vol. 546, Article ID 124087, 2020.

[41] H. Ahmad, A. R. Seadawy, T. A. Khan, and P. Thounthong, "Analytic approximate solutions for some nonlinear Parabolic 
dynamical wave equations," Journal of Taibah University for Science, vol. 14, no. 1, pp. 346-358, 2020.

[42] A. M. Aly, Z. A. S. Raizah, and M. Sheikholeslami, "Analysis of mixed convection in a sloshing porous cavity filled with a nanofluid using ISPH method," Journal of Thermal Analysis and Calorimetry, vol. 139, no. 3, pp. 1977-1991, 2020.

[43] Y. J. Zhuang and Q. Y. Zhu, "Analysis of entropy generation in combined buoyancy-Marangoni convection of power-law nanofluids in 3D heterogeneous porous media," International Journal of Heat and Mass Transfer, vol. 118, pp. 686-707, 2018.

[44] Z. A. S. Raizah, S. E. Ahmed, and A. M. Aly, "ISPH simulations of natural convection flow in E-enclosure filled with a nanofluid including homogeneous/heterogeneous porous media and solid particles," International Journal of Heat and Mass Transfer, vol. 160, Article ID 120153, 2020.

[45] M. Corcione, "Empirical correlating equations for predicting the effective thermal conductivity and dynamic viscosity of nanofluids," Energy Conversion and Management, vol. 52, no. 1, pp. 789-793, 2011.

[46] F. Garoosi, G. Bagheri, and F. Talebi, "Numerical simulation of natural convection of nanofluids in a square cavity with several pairs of heaters and coolers (HACs) inside," International Journal of Heat and Mass Transfer, vol. 67, pp. 362-376, 2013.

[47] V. Bianco, O. Manca, and S. Nardini, "Entropy generation analysis of turbulent convection flow of Al2O3-water nanofluid in a circular tube subjected to constant wall heat flux," Energy Conversion and Management, vol. 77, pp. 306-314, 2014.

[48] C. Beckermann, S. Ramadhyani, and R. Viskanta, "Natural convection flow and heat transfer between a fluid layer and a porous layer inside a rectangular enclosure," Journal of Heat Transfer, vol. 109, no. 2, pp. 363-370, 1987. 